\title{
Using Pharmacy Data and Adherence to Define Long-Term Bisphosphonate Exposure in Women
}

\author{
Monika A. Izano, PhD, MS, MA; Romain Neugebauer, PhD; Bruce Ettinger, MD; Rita Hui, PharmD, MS; \\ Malini Chandra, MS, MBA; Annette L. Adams, PhD, MPH; Fang Niu, MS; Susan M. Ott, MD; \\ and Joan C. Lo, MD
}

\section{ABSTRACT}

BACKGROUND: Assigning drug exposure is a necessary first step in examining bisphosphonate (BP) treatment in observational studies using pharmacy data.

OBJECTIVE: To determine whether the choice of adherence level using the proportion of days covered (PDC) affected BP exposure assignment.

METHODS: 10,381 female health plan members who initiated oral BP therapy between 2002 and 2010 and had received 5 consecutive years of treatment were identified and subsequently followed up to 5 additional years. In each 90-day interval of follow-up, a woman was considered "on treatment" if she received the drug for more than a predetermined PDC based on pharmacy days supply and "off treatment" if she received the drug for less than that PDC. Women who continued on therapy above the PDC threshold during follow-up were considered continuously on therapy. Women who were off treatment during the first 90-days of follow-up were classified as off therapy and were followed to determine if they remained continuously off treatment. This study evaluated the extent to which varying the PDC threshold ( $\geq 0.5, \geq 0.6$, and $\geq 0.7$ ) affected the proportion of women classified as "continuously on" or "continuously off" BP during follow-up.

RESULTS: Under PDC thresholds of $0.5,0.6$, and $0.7,48 \%, 43 \%$, and $36 \%$ of women who remained on follow-up were categorized as continuously on treatment at year 2 of follow-up, and $18 \%, 14 \%$, and $12 \%$ were categorized as continuously on treatment by the end of follow-up. Using these same PDC thresholds, $9 \%, 12 \%$, and $15 \%$ of women were categorized as off therapy during the first quarter of follow-up and were highly likely to remain off therapy: $4 \%, 5 \%$, and $5 \%$ were classified as continuously off therapy at year 2 , and $4 \%$ of women were classified as such by the end of follow-up for all 3 thresholds.

CONCLUSIONS: A PDC of 0.6 was chosen as a practical threshold for drug adherence. Varying the PDC to 0.5 or 0.7 resulted in modest changes in the proportions of women considered continuously on BP therapy.

J Manag Care Spec Pharm. 2019;25(6):719-23

Copyright $\odot 2019$, Academy of Managed Care Pharmacy. All rights reserved.

\section{What is already known about this subject}

One way of characterizing drug exposure in observational studies is to determine the proportion of a specified time interval covered by the dispensed days supply of prescriptions (proportion of days covered [PDC]).

The choice of PDC threshold may be an important determinant of which study participants are considered to remain on treatment.
The effect of setting specific PDC thresholds in characterizing exposure to oral bisphosphonate (BP) drugs in studies of longterm users (over 5 years) is not well understood.

\section{What this study adds}

Among long-term BP users, changing the PDC threshold between 0.5 and 0.7 had modest effect on the characterization of longterm exposure

BP discontinuation appears common even after 5 years of relative adherence.

Since 2008, BP use beyond the initial 5 years of treatment is less common than in previous years.

A ccurate characterization of exposure to a drug under study is a key element of drug effect evaluations in pharmacoepidemiology. The estimation of treatment effects from electronic health records (EHR) in observational studies has typically relied on the aggregation of daily participant history into discrete time intervals of equal length. ${ }^{1}$ For each time interval, researchers must decide whether a participant is taking the drug in question. One way to assess treatment adherence is to determine whether the gap between 2 consecutive prescription refills is smaller than a predetermined number of days. ${ }^{2}$ Another approach for characterizing drug exposure is to determine what proportion of a specified time interval was covered by the dispensed days supply of prescriptions. The latter is known as proportion of days covered (PDC), ${ }^{3}$ an approach similar to medication possession ratio, except that the focus for PDC is on proportion of days covered, rather than the ratio of pills dispensed to the time period covered. Observational studies that separate subjects into discrete exposed or unexposed categories must set a threshold definition of exposure. In this study, using EHR data, the effect of setting specific PDC thresholds in categorizing exposure to oral bisphosphonate (BP) drugs was evaluated.

Second-generation BP drugs are first-line therapies for osteoporosis, based on strong clinical trial evidence showing substantial osteoporotic fracture risk reduction within the first 3-4 years of treatment in high-risk patients. ${ }^{4,5}$ However, clinical trial data addressing the benefits and harms of longer exposure remain very limited. In preparation for a retrospective cohort 
study of women receiving BP therapy for more than 5 years, various algorithms for assigning exposure to an oral BP drug during follow-up were examined.

\section{Methods}

\section{Study Design and Study Population}

This retrospective observational cohort study used pharmacy health plan data within Kaiser Permanente Northern California (KNPC), a large, integrated health care delivery system providing care to approximately 4 million members.

Pharmacy records were used to identify a source population that comprised all women who (a) initiated oral BP treatment with alendronate, risedronate, or ibandronate between the years 1997 and 2005; (b) were aged 45 years or older at the time of BP treatment initiation; (c) had health plan membership within the 2 years previous to BP initiation; (d) were at least $60 \%$ adherent based on the days supply of dispensed prescriptions for each of 5 consecutive years after BP initiation; and (e) had membership gaps of no greater than 3 months during the 5 years of BP treatment. Inclusion in the study population further required evidence of $\mathrm{BP}$ treatment within the 60 days before the index date (defined below).

Women were excluded if they had received treatment with intravenous BP, etidronate, denosumab, and teriparatide; had advanced chronic kidney disease (stage 4 or 5 based on estimated glomerular filtration rate calculated using serum creatinine); received dialysis or kidney transplant; or had a diagnosis of any of the following conditions: metastatic cancer, multiple myeloma, Paget's disease, osteogenesis imperfecta, hypophosphatasia, or primary hyperparathyroidism.

Follow-up for the current study population started after 5 years of adherent BP treatment (between the years 2002 and 2010); the start of follow-up is referred to as the index date. Women were followed from their index date until (a) they died ( $n=34)$; (b) their health plan membership ended ( $n=42)$; (c) they experienced 1 of the aforementioned exclusion events $(n=43)$; or (d) they reached the study end in September 2015 $(n=10,296)$. The study was approved by the KPNC Institutional Review Board, and a waiver of informed consent was obtained due to the nature of the study.

\section{Exposure Assessment}

Ongoing BP treatment for each quarter of follow-up (90-day interval) was determined using the days supply of the dispensed drug, where stockpiling of the drug was allowed for dispensed prescriptions that overlapped 30 days or less; the later prescription was used to calculate drug supply for overlap exceeding 30 days, as previously described. ${ }^{6} \mathrm{~A}$ woman was considered "on treatment" in a follow-up quarter if she evidenced a PDC greater than a predetermined threshold. ${ }^{7}$ These data defined 2 different treatment categories: women were considered to be on treatment while they remained continuously classified as on a BP, whereas women were considered to be off treatment if they discontinued during the first quarter of follow-up and then remained continuously classified as off BP. Women who switched treatment behavior during followup were subsequently no longer in either group. For example, a hypothetical study participant who discontinued BP therapy in the first quarter of follow-up and resumed treatment in the fourth quarter would be continuously off BP for the first 3 quarters of follow-up. The participant would be in neither treatment category from the fourth quarter forward. To evaluate implications of the PDC threshold selection on treatment classification, the proportion of women classified as on BP during follow-up was examined for 3 PDC thresholds: 0.5, 0.6, and 0.7. These thresholds were chosen based on BP adherence typically observed in real-world settings among patients demonstrating persistent use. ${ }^{6,8}$

In the years following 2007, attention was directed at published data suggesting that treatment continuation beyond 5 years may not additionally benefit all patients. ${ }^{9-13}$ Concerns were also raised pertaining to rare adverse side effects occurring in patients with long-term BP exposure. ${ }^{14-17}$ To determine whether treatment continuation was affected in these later years, possible secular trends in adherence were evaluated by comparing women with index dates between 2002 and 2007 (early initiators) to those entering the cohort after 2007 (late initiators)

Analytical datasets were created in SAS version 9.3 (SAS Institute, Cary, NC) using MSMStructure. ${ }^{7}$ All analyses were performed in the R programing language, version 3.3.2. ${ }^{18}$

\section{Results}

The study population included 10,381 women, whose median age was 73 years (interquartile range: $65-79$ years); $68 \%$ of the women were white; $21 \%$ were Asian/Pacific Islander; $7 \%$ were Hispanic; and 3\% were black. Race/ethnicity was "other" or unknown for $1.2 \%$. Within this cohort, $49 \%$ of the women entered the study (after 5 years of adherent BP treatment) between the years 2002 and 2007, and 51\% entered after 2007.

Figure 1 shows the proportion of women among those who remained on follow-up and who were categorized as continuously on or continuously off BP, by PDC threshold. The largest reduction in the proportion of women who continued treatment was seen in the first 2 years of follow-up (those with total exposure of 7 years). As expected, the proportion of women on treatment was highest for 0.5 PDC and lower as the PDC threshold increased: In year 2 of follow-up, $48 \%$ of the women were categorized as being on treatment under the 0.5 PDC threshold, compared with $43 \%$ and $36 \%$ of women under the 0.6 and 0.7 PDC thresholds, respectively. 

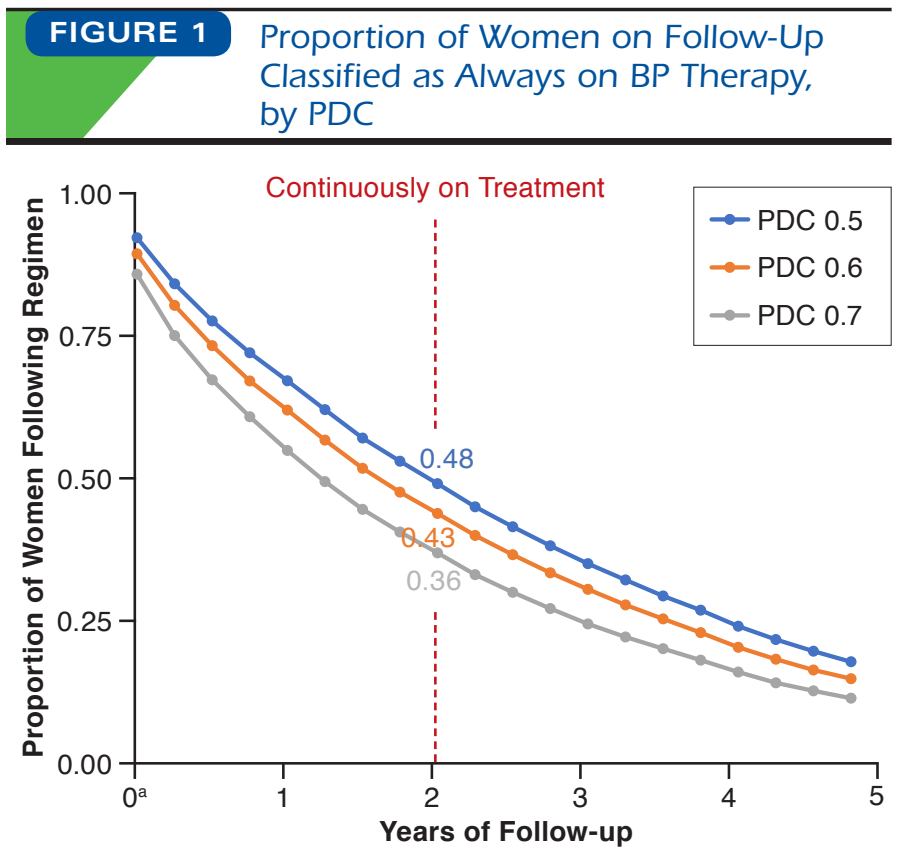

ayear 0 denotes the end of the first 90-day time interval of follow-up. The data points in year 2 (vertical line) indicate the proportion of women remaining in each treatment group.

$B P=$ bisphosphonate $P D C=$ proportion of days covered

During the first quarter of follow-up, $9 \%$ of women on follow-up were defined as being off treatment using the 0.5 threshold, $12 \%$ using the 0.6 threshold, and 15\% using the 0.7 threshold. The proportion of women categorized as remaining continuously off treatment in year 2 of follow-up varied minimally using the various PDC thresholds-0.5 (4\%), $0.6(5 \%)$, and $0.7(5 \%)$. Under all 3 thresholds, the proportion of women following the continuously off treatment category was similar at year 2 and at the study end, suggesting that those who discontinued during the first quarter of follow-up (after 5 years of treatment) were unlikely to resume taking a BP drug.

Figure 2 shows the proportion of women categorized as following each treatment arm under the 0.5 PDC threshold, among women whose index dates were between 2002 and 2007 (early initiators) versus those whose index dates were 2008 or later (late initiators). Early initiators were more likely to remain on treatment than late initiators. For instance, in year 2 of follow-up, 54\% of the early initiators remained on treatment compared with $43 \%$ of late initiators.

\section{Discussion}

Among women receiving long-term osteoporosis therapy at KPNC, changing the 0.6 PDC threshold to 0.5 or 0.7 did not greatly affect the proportion of women categorized as being continuously on or off BPs. These findings are likely due to relatively
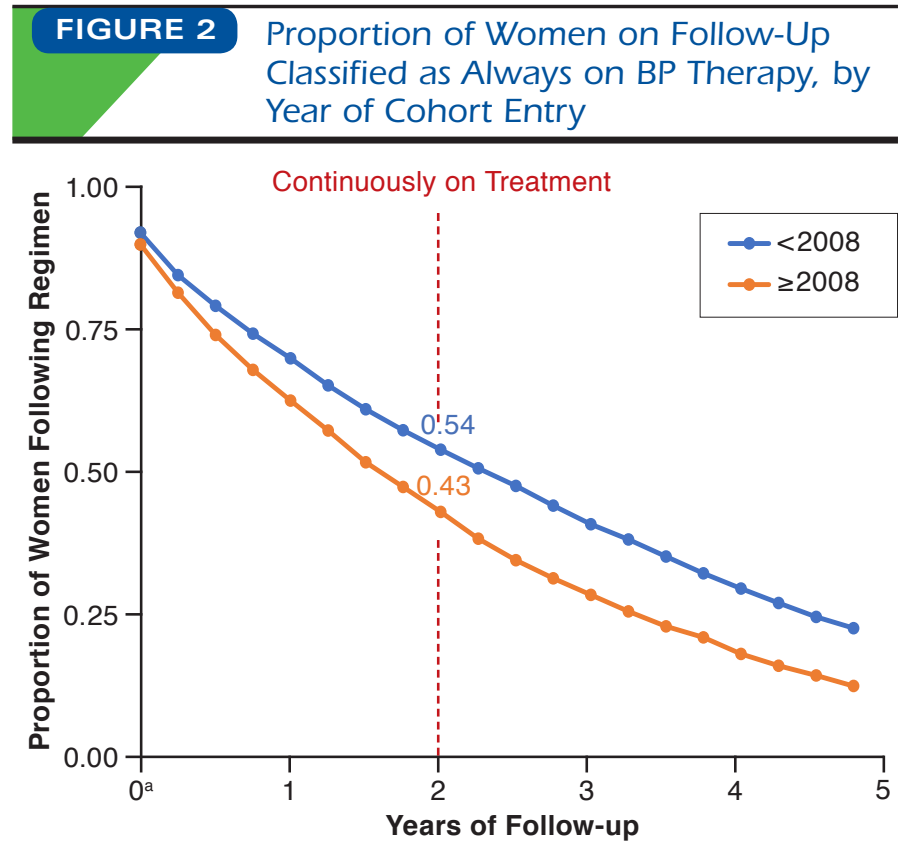

Note: The PDC cutoff was set to 0.5 .

aYear 0 denotes the end of the first 90-day time interval of follow-up. The data points in year 2 (vertical line) indicate the proportion of women remaining in each treatment group.

$B P=$ bisphosphonate; $P D C=$ proportion of days covered.

consistent treatment behaviors within this long-term treatment cohort. The differences among various PDCs were also quite small for women categorized as continuously off treatment.

The reduction in the proportion of women continuing treatment beyond 5 years of BP therapy was more gradual than previously observed among women during the first few years following BP initiation. 2,6,19 Typically, in the first year after starting oral BP therapy, a large proportion of women show low adherence and nonpersistence. $2,6,20,21$

Temporal patterns were also observed in this study. Compared with women who entered the study cohort between 2002 and 2007, women who entered in 2008 or later were more likely to not continue adherent therapy. It is possible that changing guidelines in osteoporosis affecting provider practice or awareness about risks versus benefits of long-term BP exposure may have contributed to this secular trend, 9-13,22,23 although the actual risk for late adverse complications (atypical femur fracture and osteonecrosis of the jaw) appears to be low.

\section{Limitations}

This study has a few potential limitations. First, health plan pharmacy dispensing records were used to determine drug exposure, which may not completely represent some participants' BP use, and patients may not necessarily take all the pills they receive. Nonetheless, KPNC centralized 
pharmacy databases provided complete capture of all health plan dispensed prescriptions, and detailed algorithms were used to quantify the days supply for various BP regimen types, including daily, weekly, and monthly pills and liquid formulations. Second, women considered off treatment may have received some BP drugs, but below a specified adherence threshold. Third, drug holidays were not examined, including whether patients restarted medication after treatment discontinuation. However, prolonged treatment gaps or holidays are unlikely to have affected the primary results of this study, which focused on BP exposure assignment using different thresholds to classify adherence. Finally, the study cohort was restricted to women receiving BP therapy and thus may not be generalizable to the population of men receiving osteoporosis pharmacotherapy.

The large number of women available for the reported analyses is a major strength of this study. The source population included all women enrolled at KPNC, which comprises a representative sample of the region. The goal of these analyses was to assess BP treatment behaviors after the first 5 years, a population that is growing but has been understudied. As such, understanding approaches for assessing ongoing drug exposure and adherence in the women who have already received several years of therapy is important. The cohort selection also focused on older women with a minimum $60 \%$ adherence to BP therapy for 5 years after initiation. While others might feel that an adherence of $80 \%$ would have been optimal, a lower adherence threshold of $60 \%$ assessed annually for the 5 years before cohort entry focused analyses on data that will inform osteoporosis care in real-world settings, rather than limiting attention to an unusually compliant user group.

\section{Conclusions}

This study is among the first to evaluate BP continuation and discontinuation after an initial 5 years of treatment in clinical settings. Despite adherent use over 5 years, BP nonadherence (or cessation) consistently occurred over time, and the proportion of women adhering to BPs declined over time, with about half appearing to be adherent after 2 additional years. Furthermore, changing the criteria for PDC had little effect on long-term exposure assignment among long-term BP users. It is also notable that after 2007, BP use beyond the initial 5 years of treatment appears to be less common than in the previous years. Future studies investigating this observed secular trend, possible underlying reasons at the patient or provider level, and effect on fracture outcomes are warranted.

\section{Authors}

MONIKA A. IZANO, PhD, MS, MA, Division of Research, Kaiser Permanente Northern California, Oakland, and Department of Obstetrics/Gynecology and Reproductive Sciences, University of California, San Francisco. ROMAIN NEUGEBAUER, PhD; BRUCE ETTINGER, MD; MALINI CHANDRA, MS, MBA; and JOAN C. LO, MD, Division of Research, Kaiser Permanente Northern California, Oakland. RITA HUI, PharmD, MS, Pharmacy Outcomes Research Group, Kaiser Permanente California, Oakland, and FANG NIU, MS, Pharmacy Outcomes Research Group, Kaiser Permanente California, Downey. ANNETTE L. ADAMS, PhD, MPH,

Department of Research \& Evaluation, Kaiser Permanente Southern California, Pasadena, and SUSAN M. OTT, MD, Department of Medicine, University of Washington, Seattle.

AUTHOR CORRESPONDENCE: Joan C. Lo, MD, Division of Research, Kaiser Permanente Northern California, 2000 Broadway, Oakland, CA 94612. Tel.: 510.891.3492; E-mail: Joan.C.Lo@kp.org.

\section{DISCLOSURES}

This study was supported by a grant from the National Institute of Aging and National Institute of Arthritis, Musculoskeletal and Skin Diseases at the National Institutes of Health (R01AG047230, S1). Lo has received previous research funding from Amgen and Sanofi, outside of the current study. Chandra has received previous research funding from Amgen outside of the current study. Adams has received previous research funding from Merck, Amgen, Otsuka, and Radius Health, outside of the current study. Ott previously attended a scientific advisory meeting for Amgen but declined the honorarium. Ettinger previously served as an expert witness for Teva Pharmaceuticals.

\section{REFERENCES}

1. Sofrygin O, Zhu Z, Schmittdiel JA, et al. Targeted learning with daily EHR data. arXiv.org. December 14, 2018. Available at: https://arxiv.org/ abs/1705.09874. Accessed April 23, 2019.

2. Lo JC, Pressman AR, Omar MA, Ettinger B. Persistence with weekly alendronate therapy among postmenopausal women. Osteoporos Int. 2006;17(6):922-28.

3. Nau DP. Proportion of days covered (PDC) as a preferred method of measuring medication adherence. 2015. Available at: http://ep.yimg.com/ty/cdn/ epill/pdcmpr.pdf. Accessed April 23, 2019.

4. Bilezikian JP. Efficacy of bisphosphonates in reducing fracture risk in postmenopausal osteoporosis. Am J Med. 2009;122(2 Suppl):S14-21.

5. Black DM, Cummings SR, Karpf DB, et al. Randomised trial of effect of alendronate on risk of fracture in women with existing vertebral fractures. Fracture Intervention Trial Research Group. Lancet. 1996;348(9041):1535-41.

6. Hui RL, Adams AL, Niu F, et al. Predicting adherence and persistence with oral bisphosphonate therapy in an integrated health care delivery system. J Manag Care Spec Pharm. 2017;23(4):503-12. Available at: https://www. jmcp.org/doi/pdf/10.18553/jmcp.2017.23.4.503.

7. Kaiser Permenante Division of Research. Software for causal inference research. MSMstructure. 2016. Available at: https://divisionofresearch.kaiserpermanente.org/projects/biostatistics/Pages/cisoftware.aspx. Accessed May 15, 2019.

8. Adams AL, Adams JL, Raebel MA, et al. Bisphosphonate drug holiday and fracture risk: a population-based cohort study. J Bone Miner Res. 2018;33(7):1252-59 
9. Baillie C. Continuing alendronate for an additional 5 years maintained bone mineral density in postmenopausal women. Evid Based Med. 2007;12(3):70.

10. Delmas PD. Use of alendronate after 5 years of treatment. JAMA. 2007;297(18):1979-80; author reply 1980-81.

11. Erviti J, Gorricho J. Use of alendronate after 5 years of treatment. JAMA. 2007;297(18):1979; author reply 1980-81.

12. Kraenzlin ME, Meier C. Does continued alendronate therapy improve bone mineral density and reduce fracture risk in postmenopausal women? Nat Clin Pract Endocrinol Metab. 2007;3(10):686-87.

13. Black DM, Schwartz AV, Ensrud KE, et al. Effects of continuing or stopping alendronate after 5 years of treatment: the Fracture Intervention Trial Longterm Extension (FLEX): a randomized trial. JAMA. 2006;296(24):2927-38.

14. Lo JC, O'Ryan FS, Gordon NP, et al. Prevalence of osteonecrosis of the jaw in patients with oral bisphosphonate exposure. J Oral Maxillofac Surg. 2010;68(2):243-53.

15. Khosla S, Burr D, Cauley J, et al. Bisphosphonate-associated osteonecrosis of the jaw: report of a task force of the American Society for Bone and Mineral Research. J Bone Miner Res. 2007;22(10):1479-91.

16. Shane E, Burr D, Ebeling PR, et al. Atypical subtrochanteric and diaphyseal femoral fractures: report of a task force of the American Society for Bone and Mineral Research. J Bone Miner Res. 2010;25(11):2267-94.
17. Ott SM. Long-term safety of bisphosphonates. J Clin Endocrinol Metab. 2005;90(3):1897-99.

18. R Core Team. R: a language and environment for statistical computing. R Foundation for Statistical Computing. Vienna, Austria. 2014. Available at: http://www.R-project.org/. Accessed April 23, 2019.

19. Imaz I, Zegarra P, Gonzalez-Enriquez J, Rubio B, Alcazar R, Amate JM. Poor bisphosphonate adherence for treatment of osteoporosis increases fracture risk: systematic review and meta-analysis. Osteoporos Int. 2010;21(11):1943-51.

20. Kothawala P, Badamgarav E, Ryu S, Miller RM, Halbert RJ. Systematic review and meta-analysis of real-world adherence to drug therapy for osteoporosis. Mayo Clin Proc. 2007;82(12):1493-501.

21. Lo CW, Paris PW, Holick MF. Indian and Pakistani immigrants have the same capacity as Caucasians to produce vitamin D in response to ultraviolet irradiation. Am J Clin Nutr. 1986;44(5):683-85.

22. Schwartz AV, Bauer DC, Cummings SR, et al. Efficacy of continued alendronate for fractures in women with and without prevalent vertebral fracture: the FLEX trial. J Bone Miner Res. 2010;25(5):976-82.

23. Whitaker M, Guo J, Kehoe T, Benson G. Bisphosphonates for osteoporosis—where do we go from here? N Engl J Med. 2012;366(22):2048-51. 